\title{
Iron and Neurodegeneration: From Cellular Homeostasis to Disease
}

\author{
Liliana Batista-Nascimento, Catarina Pimentel, \\ Regina Andrade Menezes, and Claudina Rodrigues-Pousada \\ Instituto de Tecnologia Química e Biológica, Universidade Nova de Lisboa, EAN, Avenida da República, 2781-901 Oeiras, Portugal \\ Correspondence should be addressed to Claudina Rodrigues-Pousada, claudina@itqb.unl.pt
}

Received 10 February 2012; Revised 21 March 2012; Accepted 5 April 2012

Academic Editor: Marcos Dias Pereira

Copyright (C) 2012 Liliana Batista-Nascimento et al. This is an open access article distributed under the Creative Commons Attribution License, which permits unrestricted use, distribution, and reproduction in any medium, provided the original work is properly cited.

\begin{abstract}
Accumulation of iron $(\mathrm{Fe})$ is often detected in the brains of people suffering from neurodegenerative diseases. High Fe concentrations have been consistently observed in Parkinson's, Alzheimer's, and Huntington's diseases; however, it is not clear whether this Fe contributes to the progression of these diseases. Other conditions, such as Friedreich's ataxia or neuroferritinopathy are associated with genetic factors that cause Fe misregulation. Consequently, excessive intracellular Fe increases oxidative stress, which leads to neuronal dysfunction and death. The characterization of the mechanisms involved in the misregulation of Fe in the brain is crucial to understand the pathology of the neurodegenerative disorders and develop new therapeutic strategies. Saccharomyces cerevisiae, as the best understood eukaryotic organism, has already begun to play a role in the neurological disorders; thus it could perhaps become a valuable tool also to study the metalloneurobiology.
\end{abstract}

\section{Iron Neurotoxicity}

Iron $(\mathrm{Fe})$ is the most important element for almost all types of cells, including brain cells. It is an essential cofactor for many proteins involved in the normal function of neuronal tissues, such as the non-heme Fe enzyme tyrosine hydroxylase required for the synthesis of myelin and the neurotransmitters dopamine, norepinephrine, and serotonin [1]. In a normal brain, $\mathrm{Fe}$ appears widely distributed by region and cell-type and it accumulates progressively during aging and neurodegenerative processes [2]. Fe is an originator of reactive oxygen species (ROS). Ferric iron $\left(\mathrm{Fe}^{3+}\right)$ can be reduced to ferrous iron $\left(\mathrm{Fe}^{2+}\right)$ by the superoxide radical $\left(\mathrm{O}_{2}^{*^{-}}\right)\left(\mathrm{Fe}^{3+}+\mathrm{O}_{2}^{\bullet^{-}} \rightarrow \mathrm{Fe}^{2+}+\mathrm{O}_{2}\right) \cdot \mathrm{Fe}^{2+}$ can also react with $\mathrm{H}_{2} \mathrm{O}_{2}$ generating the highly reactive hydroxyl free radical $(\cdot \mathrm{OH})\left(\mathrm{Fe}^{2+}+\mathrm{H}_{2} \mathrm{O}_{2} \rightarrow \mathrm{Fe}^{3+}+\cdot \mathrm{OH}+\mathrm{OH}^{-}\right.$, Fenton reaction) [3]. The combination of these reactions results in the so-called Haber-Weiss reaction $\left(\mathrm{O}_{2}^{\bullet^{-}}+\mathrm{H}_{2} \mathrm{O}_{2} \rightarrow \mathrm{O}_{2}+\right.$ $\cdot \mathrm{OH}+\mathrm{OH}^{-}$), which together with dopamine oxidation can trigger neurotoxicity [4]. Therefore, the control of Fe homeostasis is essential to keep a healthy brain.
1.1. Iron Homeostasis. In mammals, the regulatory mechanism for Fe homeostasis is mediated by the iron-regulatory proteins IRP1 and IRP2, which posttranscriptionally modulate the expression of specific mRNAs in response to intracellular $\mathrm{Fe}[5,6]$, mainly transferrin (Tf) and ferritin. Tf is an Fe-binding blood plasma glycoprotein that controls the level of free $\mathrm{Fe}$, and ferritin is an Fe storage protein composed of $\mathrm{H}$ and $\mathrm{L}$ subunits that assemble to form a hollow sphere in which ferric iron $\left(\mathrm{Fe}^{3+}\right)$ precipitates are sequestered [7]. The ferritin subunits have different functions. The $\mathrm{H}$ chains are involved in the rapid oxidation of $\mathrm{Fe}^{2+}$ to $\mathrm{Fe}^{3+}$, and the $\mathrm{L}$ chains function in the nucleation of $\mathrm{Fe}^{3+}$ within the protein shell. While the L-rich ferritins are associated with iron storage, the $\mathrm{H}$-chain ferritins are associated with responses to stress [8].

In Fe-depleted cells, IRPs bind to the IREs cis-elements in the $5^{\prime}$ UTR (untranslated region) of ferritin and in the $3^{\prime}$ UTR of TfR 1 . By binding to the IRE in the $5^{\prime}$ UTR, of ferritin, IRPs prevent translation, whereas by binding to IRE in the $3^{\prime} \mathrm{UTR}$ of TfR1, the IRPs protect the transcript from degradation [9]. In Fe-replete cells, IRPs do not bind to IREs, and 
ferritin and other transcripts are freely translated, whereas TfR 1 undergoes cleavage and subsequently degradation $[6$, $10,11]$.

Although Fe metabolism in mammals is mainly regulated at the level of absorption, changes in gene expression in response to $\mathrm{Fe}$ overload have been observed in a variety of eukaryotes from yeast to mammals [12, 13].

Ferroportin (Fpn), the basolateral membrane Fe exporter, is the only Fe exporter to date identified in mammals [1416]. Fpn mediates the release of the Fe in conjunction with ceruloplasmin $(\mathrm{Cp})$, which must oxidize the $\mathrm{Fe}^{2+}$ transported by $\mathrm{Fpn}$ to $\mathrm{Fe}^{3+}$ before release into the extracellular medium [17]. Fpn expression has also been detected on the blood-brain barrier (BBB) endothelial cells, neurons, oligodendrocytes, astrocytes, the choroid plexus and ependymal cells. Since Cp is essential for stabilization of Fpn, under conditions of $\mathrm{Cp}$ deficiency or malfunction $\mathrm{Fpn}$ is not expressed, which results in a decreased Fe efflux potentiating cellular Fe overload [18]. These observations indicate that $\mathrm{Cp}$ plays a major role in maintaining Fe homeostasis in the brain and in protecting it from $\mathrm{Fe}$-mediated free radical injury.

The Fe uptake pathway starts in the intestines, where $\mathrm{Fe}^{3+}$ is reduced to $\mathrm{Fe}^{2+}$ that then is transported to the blood by Fpn. In the blood, $\mathrm{Cp}$ oxidizes $\mathrm{Fe}^{2+}$ to $\mathrm{Fe}^{3+}$ and promotes its binding to the serum iron carrier, $\operatorname{Tf}[19]$. In order to enter the brain, Fe needs to cross two distinct barriers, the BBB and BCSF (blood-cerebrospinal fluid) [20]. The most common pathway for Fe transference across the BBB is through the TfRs expressed in the endothelial cells. The circulating Fe bound to $\mathrm{Tf}$ is captured by TfR, entering the brain by endocytosis and then is translocated across the endosomal membrane, probably through the divalent metal transporter 1 (DMT1) [21]. In addition to the Tf-TfR pathway, it has been suggested that the lactoferrin receptor-lactoferrin (LfRLf) pathway might also play a role in Fe transport across the BBB. $\mathrm{Fe}^{2+}$ in the cytoplasm can also be transported inside the mitochondria by mitoferrin or participate in electron exchange reactions $[22,23]$. Figure 1 summarizes the brain Fe uptake pathways.

Fe-related neurodegenerative disorders can result from both iron accumulation in specific brain regions or defects in its metabolism and/or homeostasis.

As the brain ages, Fe accumulates in regions that exhibit pathologic characteristics of Alzheimer's disease (AD) [24], Parkinson's disease (PD) [25], or Huntington's disease (HD) $[26,27]$. In younger individuals, the largest amounts of $\mathrm{Fe}$ are in the oligodendrocytes whereas in older individuals over 60 years old most of the Fe is found in the microglia and astrocytes of the cortex, cerebellum, hippocampus, basal ganglia, and amygdala [27]. In these regions $\mathrm{Fe}$ is either bound to neuromelanin, a dark brown pigment that accumulates essentially iron, or to ferritin [28]. Interestingly, neurons express mostly $\mathrm{H}$-ferritin, microglia express mostly L-ferritin, and oligodendrocytes express similar amounts of both subunits $[29,30]$. Additionally neurons excrete the nonrequired Fe through the carrier Fpn (Figure 1).

It has been widely accepted that abnormal high concentrations of Fe contribute to neurodegenerative processes; however, a major question has not yet been answered. Is the excessive Fe accumulation in the brain an initial event that causes neurodegeneration or a consequence of the disease process?

Fe accumulation has been shown to lead to neuronal death [31]. Available Fe interacts with molecular oxygen and generates reactive oxygen species (ROS) through Fenton and Haber-Weiss reactions [32, 33], which leads to oxidative stress. Mitochondrial dysfunction has also been raised as a common cause for a number of neurodegenerative diseases. Since mitochondria has an important role in the Fe-S clusters formation [34], malfunction can result in a low Fe-S cluster synthesis and consequent activation of DMT1 and decrease of Fpn1, Fe accumulation, and oxidative stress [4]. Oxidative injury induces lipid peroxidation, nucleic acid modification, protein misfolding and aggregation, and cell dysfunction and death [35].

1.2. Alzheimer's Disease ( $A D)$. $\mathrm{AD}$ is the most common cause of age-related neurodegeneration and is characterized by the progressive loss of memory, task performance, speech, and recognition of people and objects. $\mathrm{AD}$ is characterized by the accumulation of aggregates of insoluble amyloid- $\beta$ protein $(\mathrm{A} \beta)$, and neurofibrillary tangles (NFTs) consisting of precipitates/aggregates of hyperphosphorylated tau protein [36].

In $\mathrm{AD}$, Fe accumulation has been observed in and around the amyloid senile plaques (SP) and neurofibrillary tangles (NFTs) [37]. The excessive Fe can lead to alterations in the interaction between IRPs and their IREs [38] and disruption in the sequestration and storage of Fe by ferritin [39]. Further studies have suggested that high Fe toxicity may be due to the propensity of $\mathrm{Fe}^{2+}$ to generate ROS [40], and postmortem analysis of $\mathrm{AD}$ patients' brains has revealed activation of two enzymatic indicators of cellular oxidative stress: heme oxygenase-1 (HO-1) [41] and NADPH oxidase [42]. In addition, other evidence suggests that in $\mathrm{AD}$ the Fe metabolism is disrupted. Tf is not found in the oligodendrocytes but rather trapped within senile plaques and ferritin is expressed within reactive microglial cells that are present both in and around the senile plaques [43]. A decade ago Rogers et al. [44] provided another link between iron metabolism and $\mathrm{AD}$ pathogenesis by describing the presence of an IRE in the $5^{\prime}$ UTR of the amyloid precursor protein (APP) transcript. APP $5^{\prime}$ UTR is responsive to intracellular iron levels, which regulate translation of APP holo-protein mRNA by a mechanism similar to the translation of ferritin- $\mathrm{L}$ and $-\mathrm{H}$ mRNAs via IREs in their 5'UTRs. Recently, Duce et al. [45] have described that APP is a ferroxidase that couples with Fpn to export Fe. In AD APP ferroxidase activity appears inhibited, thereby causing neuronal Fe accumulation.

1.3. Parkinson's Disease (PD). PD is a progressive disorder that manifests as tremor at rest, bradykinesia, gait abnormalities, rigidity, postural dysfunction, and loss of balance [46]. It is the most prevalent neurodegenerative disorder after $\mathrm{AD}$ affecting about $2 \%$ of people over 65 years old. $\mathrm{PD}$ is characterized by the loss of the substantia nigra dopaminergic neurons [47] and the deposition of intracellular inclusion bodies known as Lewy bodies. The principal protein 


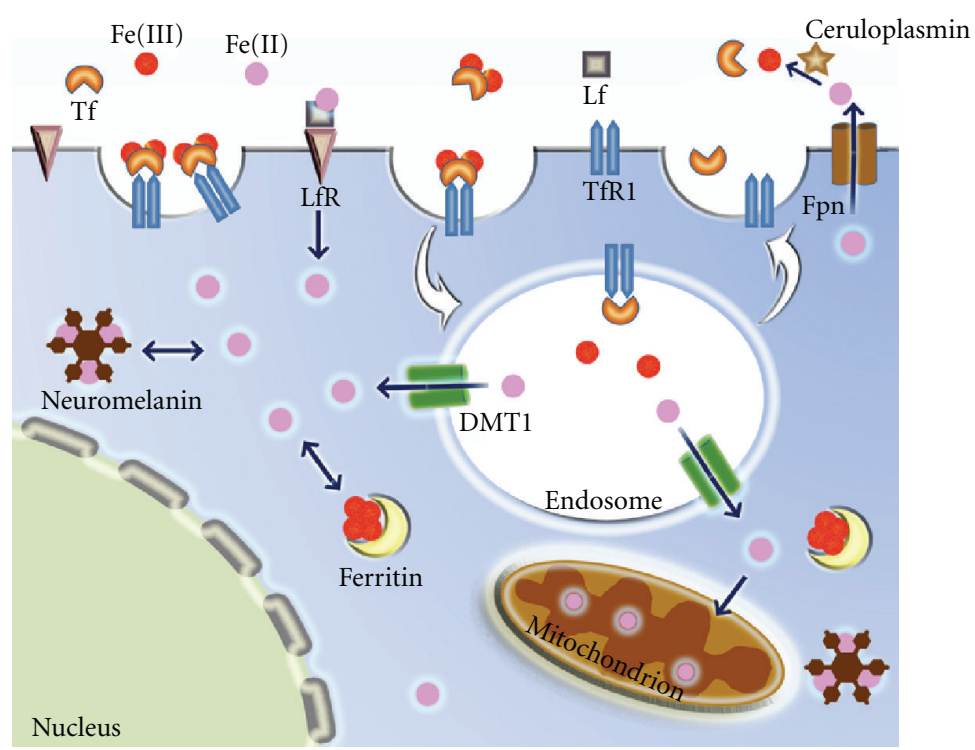

Figure 1: The brain iron homeostasis. Iron ( $\mathrm{Fe}$ ) binds to transferrin (Tf), enters the brain through the transferrin receptors (TfR) by endocytosis, and translocates across the endosomal membrane through the divalent metal transporter 1 (DMT1). Lactoferrin receptors (LfR) provide another pathway to transport Fe from Fe containing lactoferrin across the cell membranes. Inside the cell Fe binds to $\mathrm{H}$-ferritin and accumulates around the neuromelanin. Ferroportin (Fpn) transports $\mathrm{Fe}^{2+}$ outside the neuron that is oxidized to $\mathrm{Fe}^{3+}$ by ceruloplasmin promoting its binding to Tf.

component of these bodies is $\alpha$-synuclein ( $\alpha$-syn) that is ubiquitously expressed in the brain [48].

Several studies have confirmed an increase of Fe in the substantia nigra of most severe cases of PD [49-51]; however, there are still some conflicting reports about the stage during disease progression at which nigral Fe changes occur.

Nevertheless, there is a general agreement that total nigral Fe levels increase in PD, possibly leading to nigrostriatal dopamine neuron degeneration as a result of its ability to produce ROS and cause lipid peroxidation $[52,53]$.

The elevated Fe content, besides contributing to the increase of oxidative stress, also enhances $\alpha$-syn aggregates [54]. It has been shown that $\alpha$-syn harbors an IRE in its $5^{\prime}-$ UTR. Thus high intracellular Fe might also regulate $\alpha$-syn aggregation through the IRE/IRP system, therefore, causing the death of dopaminergic neurons [55]. As Fe deposits are commonly found in the Lewy bodies, Fe might play a role on the pathogenicity of $\alpha$-syn in PD.

1.4. Huntington's Disease (HD). HD is a neurodegenerative disorder characterized by progressive motor, cognitive, and psychiatric deterioration. Typically, onset of symptoms is in middle-age ( 30 and 50 years old), but the disorder can manifest at any time between infancy and elderliness. HD is caused by a dominant glutamine expansion (CAG repeat coding) within the $\mathrm{N}$-terminal of the huntingtin protein that initiates events leading to neuronal loss primarily within the striatum and cerebral cortex. Full-length huntingtin is large $(\sim 350 \mathrm{kD})$, but it is the smaller $\mathrm{N}$-terminal fragments that are the main mediators of disease progression [56]. These fragments have aberrant interactions with themselves and other biomolecules that lead to the molecular hallmarks of HD including aggregates, transcriptional repression [57], oxidative damage, and metabolic dysfunction [58].
For individuals with $\mathrm{HD}$, increased $\mathrm{Fe}$ levels have primarily been observed in the basal ganglia, namely, in the striata and the globus pallidus [59]. In addition, ferritin-Fe levels are increased in striata of early clinical HD patients as measured by magnetic resonance imaging (MRI) [60]. Fe levels increase early stage in HD and continue to increase with age, which suggests that Fe may play a role in the progression of the disease. However, the mechanisms involved in this process are not yet understood. Although both $\mathrm{AD}$ and $\mathrm{PD}$ are characterized by Fe accumulation, the Fe regulation patterns seem to be different from HD [59]. For instance, Parkinson's disease is characterized by Fe accumulation in the substantia nigra, which has not been observed in HD. It is possible that in HD Fe accumulation occurs because after neuronal loss, cells with higher Fe content replace the dead cells. Thus Fe accumulation in HD is most probably a secondary effect of the disease [60].

1.5. Other Neurological Disorders. The accumulation of Fe has also been implicated in a series of other neurological diseases, such as Neuroferritinopathy, Hallervorden-Spatz syndrome, and Aceruloplasminemia that are characterized by mutations in genes that encode for ferritin light polypeptide (FTL), pantothenate kinase (PANK2), and ceruloplasmin, respectively.

Neuroferritinopathy is dominantly inherited and is a late-onset disease of the basal ganglia that presents extrapyramidal features similar to HD and PD. It is caused by a single adenine insertion at position 460-461 that is predicted to change the C-terminal residues of the gene encoding L-chain ferritin [61]. Brain histochemistry of patients with neuroferritinopathy showed abnormal aggregates of ferritin and $\mathrm{Fe}$ and low serum ferritin concentrations. The C-terminus of the aberrant $\mathrm{L}$ chain might interfere with the formation of 
the hollow sphere allowing inappropriate release of Fe from the loaded ferritin [62].

Another evidence for the involvement of $\mathrm{Fe}$ in neurodegeneration is provided by the study of HallervordenSpatz syndrome (HSS), also referred to as neurodegeneration with brain-iron accumulation 1 (NBIA) or pantothenatekinase-2-associated neurodegeneration (PANK2) [63]. HSS is an autosomal recessive disorder characterized by dystonia, pigmentary retinopathy in children and neuropsychiatric defects in adults. The HSS patient's MRI has a characteristic pattern in the globus pallidus, known as "the eye of the tiger" because of its appearance [64]. Zhou et al. [63] identified the genetic basis for this neurodegenerative disease in which Fe accumulation is most dramatic by detecting the underlying mutations in the gene that encodes for pantothenate-kinase. This enzyme is essential for the coenzyme A biosynthesis [65], which in turn catalyzes the phosphorylation of pantothenate (vitamin $\mathrm{B}_{5}$ ), $\mathrm{N}$-pantothenoylcysteine, and pantetheine [63]. The product of this reaction, $4^{\prime}$-phosphopantothenate, is then converted to $4^{\prime}$ phosphopanthetheine in a reaction that consumes cysteine. HSS results from $4^{\prime}$-phosphopantothenate deficit, which is caused by genetic defects in PANK2. Given that cysteine is consumed in the conversion of $4^{\prime}$-phosphopantothenate, an absence of functional PANK2 might explain the observed accumulation of cysteine in the degenerating brain areas of HSS patients. Consequently the cysteine Fe-chelating properties might account for the observed regional Fe accumulation, and cysteine-bound Fe may promote Fe-dependent oxidative damage in these regions [66]. Even though PANK2 is not directly involved in Fe metabolism, its absence may contribute for Fe accumulation in the brain, leading to neuronal death via oxidative stress.

Finally, aceruloplasminemia, an autosomal recessive disorder caused by mutations in the ceruloplasmin gene, also results in Fe overload in the brain characterized mainly by retinal neurodegeneration [17]. $\mathrm{Cp}$ is a multicopper ferroxidase responsible for the Fe homeostasis by promoting $\mathrm{Fe}$ incorporation into $\mathrm{Tf}$, therefore, playing a key role in releasing Fe from the cells [67]. Consequently, mutations in the ceruloplasmin gene may cause Fe metabolism misregulation in the brain. Due to the low release of cellular Fe and the high nontransferrin-bound Fe uptake, the intracellular Fe concentration becomes abnormally high. This induces oxidative stress and formation of ROS triggering a cascade of pathological events that lead to neuronal death.

1.6. Fe-Chelation Therapies. Oxidative stress, protein aggregation, and active redox Fe have been considered promising pharmacological targets for the treatment of $\mathrm{AD}$ and $\mathrm{PD}$. $\mathrm{BBB}$ permeable Fe chelators can be used as potential therapeutic agents in the treatment of neurodegenerative diseases. A promising Fe chelator is desferrioxamine (Desferal), which has been shown to prevent up to $60 \%$ of dopaminergic neurons from death in a rat model of PD [68]. The main disadvantage of desferrioxamine is that it cannot cross the $\mathrm{BBB}$, due to its size and hydrophobicity [65]. Clioquinol, a small lipophilic Fe chelator that can cross the BBB, has also proved to have beneficial effects in patients with AD [69].
However, clioquinol is not iron selective and has very toxic effects. Aroylhydrazones are the new nontoxic lipophilic Fe chelators that can form a neutral complex with $\mathrm{Fe}$ and diffuse out of the membrane [70]. Other important class of compounds proposed for therapy is the polyphenols that have antioxidant properties and can bind Fe [71]. A major limitation is their capacity to be absorbed at the gastrointestinal tract and subsequently be transported through the BBB.

The development of an effective non-toxic therapeutic agent for such complex brain disorders still represents a challenging task.

\section{The Yeast Model}

In the last decade, the budding yeast Saccharomyces cerevisiae has been used as a model system to gain insights about the mechanisms of neurodegenerative disorders such as Parkinson's, Huntington's, and Alzheimer's [72]. Yeast cells are generally used to study key proteins involved in the etiology and/or pathology of these diseases. When a yeast homologue exists, the corresponding gene can be easily disrupted or overexpressed to determine the loss or gain of function phenotypes, respectively. When a yeast homologue is not present, the human gene can be expressed in yeast and any relevant phenotype that results from this expression can be analyzed. The latter has been called humanized yeast models [73]. Despite their simplicity, yeast cells possess most of the same basic cellular machinery as neurons in the brain, including pathways required for protein homeostasis and energy metabolism. Also their easy genetic manipulation makes these cells an ideal tool for molecular biology.

Many of the genes and biological systems that function in yeast Fe homeostasis are conserved throughout eukaryotes to humans [74]. S. cerevisiae expresses three genetically distinct transport systems for $\mathrm{Fe}$, two reductive systems and one nonreductive system. The reductive Fe uptake system consists in a low-affinity pathway defined by Fet4, that can also transport other metals and in a high-affinity pathway that is mediated by a protein complex composed of a multicopper ferroxidase Fet3, the mammalian Cp homologue, and a permease Ftr1. The Fet3-Ftr1 complex is specific for Fe and is regulated both transcriptionally and posttranscriptionally by this metal [75-77]. The nonreductive Fe uptake system is mediated by the ARN family (Arn1-4) of membrane permeases that transport siderophore- $\mathrm{Fe}^{3+}$ complexes [78, 79]. Additionally Harris et al. [80] showed for the first time that Fet3 can functionally replace ceruloplasmin in restoring Fe homeostasis.

Moreover, cells are able to spare Fe through the regulation of Tis 11 homologues and Cth1/2-mediated degradation of mRNAs coding for Fe-binding proteins, thereby facilitating the utilization of limited cellular Fe levels [81, 82].

Since $S$. cerevisiae lacks the Fe storage protein, ferritin, during $\mathrm{Fe}$ overload this is sequestered into the vacuole by the Cccl transporter, which is under the control of the Yap5 transcription factor [13]. On the other hand, Fet5/Fth1 complex mobilizes $\mathrm{Fe}$ out of the vacuole for use during $\mathrm{Fe}$ limitation [83]. 
Given the similarities between yeast and mammals and the availability of humanized $S$. cerevisiae strains, yeast could potentially become an effective model to dissect the molecular pathway associated with the misregulation of $\mathrm{Fe}$ homeostasis in the neurodegenerative diseases.

One good example of the use of yeast to study Fe accumulation in a neurodegenerative disease was first reported for Friedreich's ataxia (FRDA). FRDA is an autosomal recessive mitochondrial disorder that causes progressive damage to the nervous system, resulting in gait disturbance, speech problems, heart disease, and diabetes. It is caused by GAA triplet expansion in the first intron of the frataxin gene (FA) [84].

A gene with high sequence similarity to $F A$ was initially identified in yeast, the yeast frataxin homologue, YFH1 [85] and later it was shown that the two proteins were both located in the mitochondria. Moreover, human FA could complement for the absence of the yeast $y f h 1$ [86]. However, FA function was only discovered when Lodi and coworkers [87] showed that the YFH1 knockout strain led to an excessive Fe accumulation in the mitochondria resulting in the generation of ROS and consequently oxidative damage. The yeast frataxin homologue provided the evidence that FRDA is indeed a mitochondrial disorder. The yeast model allowed a better understanding of the FDRA pathophysiology and provided a tool for assaying therapeutic targets.

\section{Concluding Remarks}

In this paper, we have summarized the role of Fe, a redoxactive transition metal, in neurodegenerative disorders. Despite a considerable investigation already performed, it is still not clear whether excessive Fe accumulation in the brain is an initial event that causes neuronal death or is a consequence of the disease process. The growing evidence suggests that the abnormal high Fe levels in the brain may have genetic causes, as found in patients with aceruloplasminemia, or sporadic causes that can disrupt the normal mechanisms of $\mathrm{Fe}$ transport into the brain. In addition, elevated $\mathrm{Fe}$ levels generate ROS and increase the levels of oxidative stress, which is considered one of the pathways leading to neuronal death. A new study from Lei et al. [88] shows that loss of Tau impairs the Fpn Fe export by preventing the proper trafficking of APP ferroxidase to the neuronal surface, leading to $\mathrm{Fe}$ accumulation, which results in degeneration of dopaminergic neurons in PD. These findings suggest the involvement of a new mechanism associated with Tau's role in PD. However, the precise role of Fe transport proteins in the brain is not completely understood, which impairs the success of therapeutic strategies to prevent the damaging effects of the Fe in the brain.

Finally, we believe that the use of the yeast neurodegenerative disease models might provide valuable insights into key aspects of the Fe pathology in the brain and pave the way towards the discovery of promising therapeutic targets.

\section{Acknowledgments}

This paper was supported by Grants from the Fundação para a Ciência e a Tecnologia (FCT), no. SFRH/BD/39389/2007 to L. Batista-Nascimento, no. SFRH/BPD/35052/2007 to C. Pimentel, no. SFRH/BPD/26506/2006 to R. A. Menezes and no. PTDC/BIAMIC/108747/2008 and Pest-OE/EQB/ LA0004/2011 to C. Rodrigues-Pousada.

\section{References}

[1] J. L. Beard, J. A. Wiesinger, and J. R. Connor, "Pre- and postweaning iron deficiency alters myelination in spraguedawley rats," Developmental Neuroscience, vol. 25, no. 5, pp. 308-315, 2003.

[2] D. T. Dexter, J. Sian, P. Jenner, and C. D. Marsden, "Implications of alterations in trace element levels in brain in Parkinson's disease and other neurological disorders affecting the basal ganglia," Advances in Neurology, vol. 60, pp. 273-281, 1993.

[3] K. Jomova and M. Valko, "Advances in metal-induced oxidative stress and human disease," Toxicology, vol. 283, no. 2-3, pp. 65-87, 2011.

[4] M. T. Núñez, P. Urrutia, N. Mena, P. Aguirre, V. Tapia, and J. Salazar, "Iron toxicity in neurodegeneration," Biometals. In press.

[5] M. W. Hentze, M. U. Muckenthaler, and N. C. Andrews, "Balancing acts: molecular control of mammalian iron metabolism," Cell, vol. 117, no. 3, pp. 285-297, 2004.

[6] T. A. Rouault, "The role of iron regulatory proteins in mammalian iron homeostasis and disease," Nature Chemical Biology, vol. 2, no. 8, pp. 406-414, 2006.

[7] P. Ponka, C. Beaumont, and D. R. Richardson, "Function and regulation of transferrin and ferritin," Seminars in Hematology, vol. 35, no. 1, pp. 35-54, 1998.

[8] A. M. Koorts and M. Viljoen, "Ferritin and ferritin isoforms I: structure-function relationships, synthesis, degradation and secretion," Archives of Physiology and Biochemistry, vol. 113, no. 1, pp. 30-54, 2007.

[9] A. Wilczynska, C. Aigueperse, M. Kress, F. Dautry, and D. Weil, "The translational regulator CPEB1 provides a link between dcpl bodies and stress granules," Journal of Cell Science, vol. 118, no. 5, pp. 981-992, 2005.

[10] B. Galy, D. Ferring, M. Benesova, V. Benes, and M. W. Hentze, "Targeted mutagenesis of the murine IRP1 and IRP2 genes reveals context-dependent RNA processing differences in vivo," RNA, vol. 10, no. 7, pp. 1019-1025, 2004.

[11] E. C. Theil and R. S. Eisenstein, "Combinatorial mRNA regulation: iron regulatory proteins and iso-iron-responsive elements (Iso-IREs)," Journal of Biological Chemistry, vol. 275, no. 52, pp. 40659-40662, 2000.

[12] S. J. Romney, C. Thacker, and E. A. Leibold, "An Iron Enhancer Element in the FTN-1 gene directs iron-dependent expression in Caenorhabditis elegans intestine," Journal of Biological Chemistry, vol. 283, no. 2, pp. 716-725, 2008.

[13] L. Li, D. Bagley, D. M. Ward, and J. Kaplan, "Yap5 is an ironresponsive transcriptional activator that regulates vacuolar iron storage in yeast," Molecular and Cellular Biology, vol. 28, no. 4, pp. 1326-1337, 2008.

[14] S. Abboud and D. J. Haile, "A novel mammalian iron-regulated protein involved in intracellular iron metabolism," Journal of Biological Chemistry, vol. 275, no. 26, pp. 19906-19912, 2000.

[15] A. Donovan, A. Brownlie, Y. Zhou et al., "Positional cloning of zebrafish ferroportin1 identifies a conserved vertebrate iron exporter," Nature, vol. 403, no. 6771, pp. 776-781, 2000. 
[16] A. T. McKie, P. Marciani, A. Rolfs et al., "A novel duodenal iron-regulated transporter, IREG1, implicated in the basolateral transfer of iron to the circulation," Molecular Cell, vol. 5, no. 2, pp. 299-309, 2000.

[17] Z. L. Harris, A. P. Durley, T. K. Man, and J. D. Gitlin, “Targeted gene disruption reveals an essential role for ceruloplasmin in cellular iron efflux," Proceedings of the National Academy of Sciences of the United States of America, vol. 96, no. 19, pp. 10812-10817, 1999.

[18] G. J. Anderson and F. Wang, "Essential but toxic: controlling the flux of iron in the body," Clinical and Experimental Pharmacology and Physiology. In press.

[19] D. B. Kell, "Iron behaving badly: inappropriate iron chelation as a major contributor to the aetiology of vascular and other progressive inflammatory and degenerative diseases," $B M C$ Medical Genomics, vol. 2, article 2, 2009.

[20] W. M. Pardridge, J. Eisenberg, and Jing Yang, "Human bloodbrain barrier transferrin receptor," Metabolism, vol. 36, no. 9, pp. 892-895, 1987.

[21] J. R. Burdo and J. R. Connor, "Brain iron uptake and homeostatic mechanisms: an overview," BioMetals, vol. 16, no. 1, pp. 63-75, 2003.

[22] B. A. Faucheux, N. Nillesse, P. Damier et al., "Expression of lactoferrin receptors is increased in the mesencephalon of patients with Parkinson disease," Proceedings of the National Academy of Sciences of the United States of America, vol. 92, no. 21, pp. 9603-9607, 1995.

[23] G. A. Salvador, "Iron in neuronal function and dysfunction," BioFactors, vol. 36, no. 2, pp. 103-110, 2010.

[24] G. Bartzokis, D. Sultzer, J. Mintz et al., "In vivo evaluation of brain iron in alzheimer's disease and normal subjects using MRI," Biological Psychiatry, vol. 35, no. 7, pp. 480-487, 1994.

[25] G. Bartzokis, J. L. Cummings, C. H. Markham et al., "MRI evaluation of brain iron in earlier- and later-onset Parkinson's disease and normal subjects," Magnetic Resonance Imaging, vol. 17, no. 2, pp. 213-222, 1999.

[26] C. K. Jurgens, R. Jasinschi, A. Ekin et al., "MRI T2 hypointensities in basal ganglia of premanifest Huntington's disease," PLoS Currents, vol. 2, Article ID RRN1173, 2010.

[27] J. H. Duyn, "High-field MRI of brain iron," Methods in Molecular Biology, vol. 711, pp. 239-249, 2011.

[28] L. Zecca, F. A. Zucca, P. Costi et al., "The neuromelanin of human substantia nigra: structure, synthesis and molecular behaviour," Journal of Neural Transmission, Supplement, no. 65, pp. 145-155, 2003.

[29] P. M. Harrison and P. Arosio, "The ferritins: molecular properties, iron storage function and cellular regulation," Biochimica et Biophysica Acta, vol. 1275, no. 3, pp. 161-203, 1996.

[30] L. Zecca, A. Stroppolo, A. Gatti et al., "The role of iron and molecules in the neuronal vulnerability of locus coeruleus and substantia nigra during aging," Proceedings of the National Academy of Sciences of the United States of America, vol. 101, no. 26, pp. 9843-9848, 2004.

[31] Z. M. Qian and X. Shen, "Brain iron transport and neurodegeneration," Trends in Molecular Medicine, vol. 7, no. 3, pp. 103-108, 2001.

[32] B. Halliwell, "Reactive oxygen species and the central nervous system," Journal of Neurochemistry, vol. 59, no. 5, pp. 1609-1623, 1992.

[33] B. Halliwell, "Free radicals, proteins and DNA: oxidative damage versus redox regulation," Biochemical Society Transactions, vol. 24, no. 4, pp. 1023-1027, 1996.

[34] R. Lill, R. Dutkiewicz, H. P. Elsässer et al., "Mechanisms of iron-sulfur protein maturation in mitochondria, cytosol and nucleus of eukaryotes," Biochimica et Biophysica Acta, vol. 1763, no. 7, pp. 652-667, 2006.

[35] J. N. Keller, R. J. Mark, A. J. Bruce et al., "4-hydroxynonenal, an aldehydic product of membrane lipid peroxidation, impairs glutamate transport and mitochondrial function in synaptosomes," Neuroscience, vol. 80, no. 3, pp. 685-696, 1997.

[36] C. A. Ross and M. A. Poirier, "Protein aggregation and neurodegenerative disease," Nature Medicine, vol. 10, pp. S10-S17, 2004.

[37] M. A. Smith, P. L. R. Harris, L. M. Sayre, and G. Perry, "Iron accumulation in Alzheimer disease is a source of redoxgenerated free radicals," Proceedings of the National Academy of Sciences of the United States of America, vol. 94, no. 18, pp. 9866-9868, 1997.

[38] T. A. Fulga, I. Elson-Schwab, V. Khurana et al., "Abnormal bundling and accumulation of F-actin mediates tau-induced neuronal degeneration in vivo," Nature Cell Biology, vol. 9, no. 2, pp. 139-148, 2007.

[39] D. J. Piñero, J. Hu, and J. R. Connor, "Alterations in the interaction between iron regulatory proteins and their iron responsive element in normal and Alzheimer's diseased brains," Cellular and Molecular Biology, vol. 46, no. 4, pp. 761-776, 2000.

[40] K. Jomova and M. Valko, "Importance of iron chelation in free radical-induced oxidative stress and human disease," Current Pharmaceutical Design, vol. 17, no. 31, pp. 3460-3473, 2011.

[41] A. Takeda, M. A. Smith, J. Avilá et al., "In Alzheimer's disease, heme oxygenase is coincident with Alz50, an epitope of $\tau$ induced by 4-hydroxy-2-nonenal modification," Journal of Neurochemistry, vol. 75, no. 3, pp. 1234-1241, 2000.

[42] S. Shimohama, H. Tanino, N. Kawakami et al., "Activation of NADPH oxidase in Alzheimer's disease brains," Biochemical and Biophysical Research Communications, vol. 273, no. 1, pp. 5-9, 2000.

[43] E. Grünblatt, J. Bartl, and P. Riederer, "The link between iron, metabolic syndrome, and Alzheimer's disease," Journal of Neural Transmission, vol. 118, no. 3, pp. 371-379, 2011.

[44] J. T. Rogers, J. D. Randall, C. M. Cahill et al., "An ironresponsive element type II in the 5 - -untranslated region of the Alzheimer's amyloid precursor protein transcript," Journal of Biological Chemistry, vol. 277, no. 47, pp. 45518-45528, 2002.

[45] J. A. Duce, A. Tsatsanis, M. A. Cater et al., "Iron-export ferroxidase activity of $\beta$-amyloid precursor protein is inhibited by zinc in Alzheimer's disease," Cell, vol. 142, no. 6, pp. 857-867, 2010.

[46] J. Jankovic, "Parkinson's disease: clinical features and diagnosis," Journal of Neurology, Neurosurgery and Psychiatry, vol. 79, no. 4, pp. 368-376, 2008.

[47] M. C. Irizarry, W. Growdon, T. Gomez-Isla et al., "Nigral and cortical Lewy bodies and dystrophic nigral neurites in Parkinson's disease and cortical Lewy body disease contain $\alpha$-synuclein immunoreactivity," Journal of Neuropathology and Experimental Neurology, vol. 57, no. 4, pp. 334-337, 1998.

[48] M. G. Spillantini, M. L. Schmidt, V. M. Y. Lee, J. Q. Trojanowski, R. Jakes, and M. Goedert, " $\alpha$-synuclein in Lewy bodies,” Nature, vol. 388, no. 6645, pp. 839-840, 1997.

[49] D. T. Dexter, F. R. Wells, F. Agid et al., "Increased nigral iron content in postmortem Parkinsonian brain," The Lancet, vol. 2, no. 8569, pp. 1219-1220, 1987.

[50] P. Riederer, E. Sofic, W. D. Rausch et al., "Transition metals, ferritin, glutathione, and ascorbic acid in parkinsonian brains," Journal of Neurochemistry, vol. 52, no. 2, pp. 515-520, 1989.

[51] E. C. Hirsch, J. P. Brandel, P. Galle, F. Javoy-Agid, and Y. Agid, "Iron and aluminum increase in the substantia nigra 
of patients with Parkinson's disease: an X-ray microanalysis," Journal of Neurochemistry, vol. 56, no. 2, pp. 446-451, 1991.

[52] M. B. H. Youdim, D. Ben-Shachar, and P. Riederer, "Iron in brain function and dysfunction with emphasis on Parkinson's disease," European Neurology, vol. 31, supplement 1, pp. 34-40, 1991.

[53] K. Jomova, D. Vondrakova, M. Lawson, and M. Valko, "Metals, oxidative stress and neurodegenerative disorders," Molecular and Cellular Biochemistry, vol. 345, no. 1-2, pp. 91-104, 2010.

[54] M. Hashimoto, A. Takeda, L. J. Hsu, T. Takenouchi, and E. Masliah, "Role of cytochrome c as a stimulator of $\alpha$-synuclein aggregation in Lewy body disease," Journal of Biological Chemistry, vol. 274, no. 41, pp. 28849-28852, 1999.

[55] W. Li, H. Jiang, N. Song, and J. Xie, "Oxidative stress partially contributes to iron-induced alpha-synuclein aggregation in SK-N-SH cells," Neurotoxicity Research, vol. 19, no. 3, pp. 435-442, 2011.

[56] R. K. Graham, Y. Deng, E. J. Slow et al., "Cleavage at the caspase- 6 site is required for neuronal dysfunction and degeneration due to mutant huntingtin," Cell, vol. 125, no. 6, pp. 1179-1191, 2006.

[57] M. DiFiglia, E. Sapp, K. O. Chase et al., "Aggregation of huntingtin in neuronal intranuclear inclusions and dystrophic neurites in brain," Science, vol. 277, no. 5334, pp. 1990-1993, 1997.

[58] S. E. Browne, A. C. Bowling, U. MacGarvey et al., "Oxidative damage and metabolic dysfunction in huntington's disease: selective vulnerability of the basal ganglia," Annals of Neurology, vol. 41, no. 5, pp. 646-653, 1997.

[59] G. Bartzokis, J. Cummings, S. Perlman, D. B. Hance, and J. Mintz, "Increased basal ganglia iron levels in Huntington disease," Archives of Neurology, vol. 56, no. 5, pp. 569-574, 1999.

[60] G. Bartzokis and T. A. Tishler, "MRI evaluation of basal ganglia ferritin iron and neurotoxicity in Alzheimer's and Huntingon's disease," Cellular and Molecular Biology, vol. 46, no. 4, pp. 821-833, 2000.

[61] A. R. J. Curtis, C. Fey, C. M. Morris et al., "Mutation in the gene encoding ferritin light polypeptide causes dominant adult-onset basal ganglia disease," Nature Genetics, vol. 28, no. 4, pp. 350-354, 2001.

[62] T. A. Rouault, "Iron on the brain," Nature Genetics, vol. 28, no. 4, pp. 299-300, 2001.

[63] B. Zhou, S. K. Westaway, B. Levinson, M. A. Johnson, J. Gitschier, and S. J. Hayflick, "A novel pantothenate kinase gene (PANK2) is defective in Hallervorden-Spatz syndrome," Nature Genetics, vol. 28, no. 4, pp. 345-349, 2001.

[64] S. J. Hayflick, "Unraveling the Hallervorden-Spatz syndrome: pantothenate kinase-associated neurodegeneration is the name," Current Opinion in Pediatrics, vol. 15, no. 6, pp. 572-577, 2003.

[65] N. Rakba, F. Aouad, C. Henry et al., "Iron mobilisation and cellular protection by a new synthetic chelator O- Trensox," Biochemical Pharmacology, vol. 55, no. 11, pp. 1797-1806, 1998.

[66] Y. Ke and Z. M. Qian, "Iron misregulation in the brain: a primary cause of neurodegenerative disorders," The Lancet Neurology, vol. 2, no. 4, pp. 246-253, 2003.

[67] N. E. Hellman and J. D. Gitlin, "Ceruloplasmin metabolism and function," Annual Review of Nutrition, vol. 22, pp. 439-458, 2002.

[68] C. A. Perez, Y. Tong, and M. Guo, "Iron chelators as potential therapeutic agents for Parkinson's disease," Current Bioactive Compounds, vol. 4, no. 3, pp. 150-158, 2008.
[69] Y. Wang, R. Branicky, Z. Stepanyan et al., "The antineurodegeneration drug clioquinol inhibits the agingassociated protein CLK-1," Journal of Biological Chemistry, vol. 284, no. 1, pp. 314-323, 2009.

[70] X. Li, J. Jankovic, and W. Le, "Iron chelation and neuroprotection in neurodegenerative diseases," Journal of Neural Transmission, vol. 118, no. 3, pp. 473-477, 2011.

[71] R. G. Andrade Jr., J. S. Ginani, G. K. B. Lopes, F. Dutra, A. Alonso, and M. Hermes-Lima, "Tannic acid inhibits in vitro iron-dependent free radical formation," Biochimie, vol. 88, no. 9, pp. 1287-1296, 2006.

[72] S. Tenreiro and T. F. Outeiro, "Simple is good: yeast models of neurodegeneration,” FEMS Yeast Research, vol. 10, no. 8, pp. 970-979, 2010.

[73] V. Khurana and S. Lindquist, "Modelling neurodegeneration in Saccharomyces cerevisiae: why cook with baker's yeast?" Nature Reviews Neuroscience, vol. 11, no. 6, pp. 436-449, 2010.

[74] M. R. Bleackley and R. T. A. MacGillivray, "Transition metal homeostasis: from yeast to human disease," BioMetals, vol. 24, no. 5, pp. 785-809, 2011.

[75] A. Dancis, R. D. Klausner, A. G. Hinnebusch, and J. G. Barriocanal, "Genetic evidence that ferric reductase is required for iron uptake in Saccharomyces cerevisiae," Molecular and Cellular Biology, vol. 10, no. 5, pp. 2294-2301, 1990.

[76] E. Georgatsou and D. Alexandraki, "Two distinctly regulated genes are required for ferric reduction, the first step of iron uptake in Saccharomyces cerevisiae," Molecular and Cellular Biology, vol. 14, no. 5, pp. 3065-3073, 1994.

[77] M. R. Felice, I. De Domenico, L. Li et al., "Post-transcriptional regulation of the yeast high affinity iron transport system," Journal of Biological Chemistry, vol. 280, no. 23, pp. 2218122190, 2005.

[78] C. C. Philpott and O. Protchenko, "Response to iron deprivation in Saccharomyces cerevisiae," Eukaryotic Cell, vol. 7, no. 1, pp. 20-27, 2008.

[79] T. Nevitt, "War-Fe-Re: iron at the core of fungal virulence and host immunity," BioMetals, vol. 24, no. 3, pp. 547-558, 2011.

[80] Z. L. Harris, S. R. Davis-Kaplan, J. D. Gitlin, and J. Kaplan, "A fungal multicopper oxidase restores iron homeostasis in aceruloplasminemia," Blood, vol. 103, no. 12, pp. 4672-4673, 2004.

[81] S. Puig, E. Askeland, and D. J. Thiele, "Coordinated remodeling of cellular metabolism during iron deficiency through targeted mRNA degradation," Cell, vol. 120, no. 1, pp. 99-110, 2005.

[82] S. Puig, S. V. Vergara, and D. J. Thiele, "Cooperation of two mRNA-binding proteins drives metabolic adaptation to iron deficiency," Cell Metabolism, vol. 7, no. 6, pp. 555-564, 2008.

[83] J. L. Urbanowski and R. C. Piper, "The iron transporter Fth1p forms a complex with the Fet5 iron oxidase and resides on the vacuolar membrane," Journal of Biological Chemistry, vol. 274, no. 53, pp. 38061-38070, 1999.

[84] V. Campuzano, L. Montermini, M. D. Moltò et al., "Friedreich's ataxia: autosomal recessive disease caused by an intronic GAA triplet repeat expansion," Science, vol. 271, no. 5254, pp. 1423-1427, 1996.

[85] M. Babcock, D. De Silva, R. Oaks et al., "Regulation of mitochondrial iron accumulation by Yfh1p, a putative homolog of frataxin," Science, vol. 276, no. 5319, pp. 1709-1712, 1997.

[86] P. Cavadini, C. Gellera, P. I. Patel, and G. Isaya, "Human frataxin maintains mitochondrial iron homeostasis in Saccharomyces cerevisiae," Human Molecular Genetics, vol. 9, no. 17 , pp. 2523-2530, 2000. 
[87] R. Lodi, J. M. Cooper, J. L. Bradley et al., "Deficit of in vivo mitochondrial ATP production in patients with Friedreich ataxia," Proceedings of the National Academy of Sciences of the United States of America, vol. 96, no. 20, pp. 11492-11495, 1999.

[88] P. Lei, S. Ayton, D. I. Finkelstein et al., "Tau deficiency induces parkinsonism with dementia by impairing APP-mediated iron export," Nature Medicine, vol. 18, no. 2, pp. 291-295, 2012. 


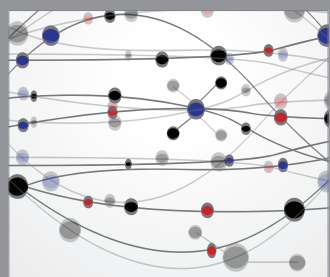

The Scientific World Journal
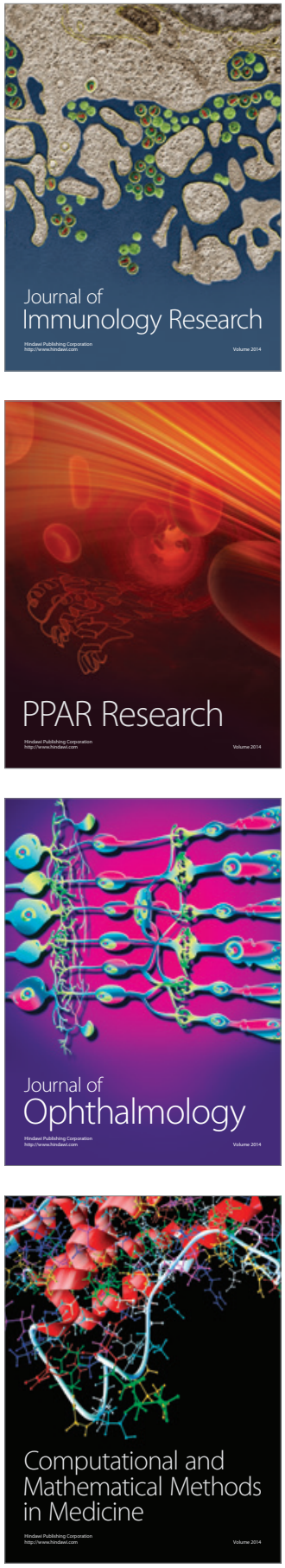

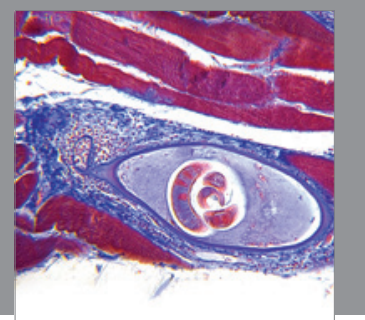

Gastroenterology

Research and Practice
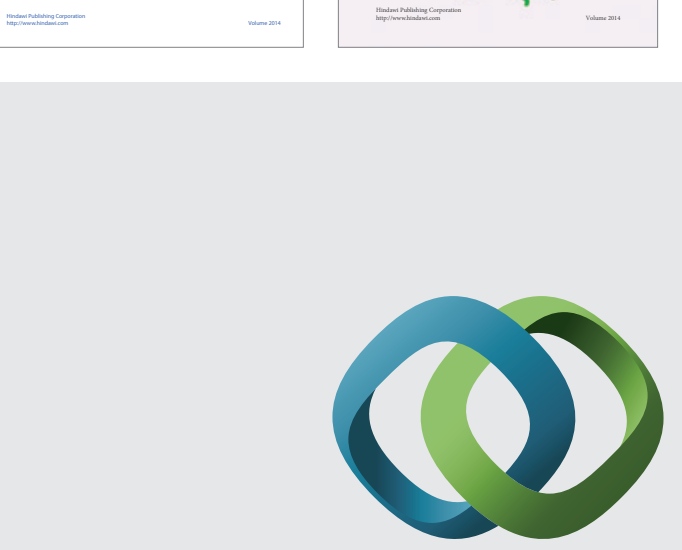

\section{Hindawi}

Submit your manuscripts at

http://www.hindawi.com
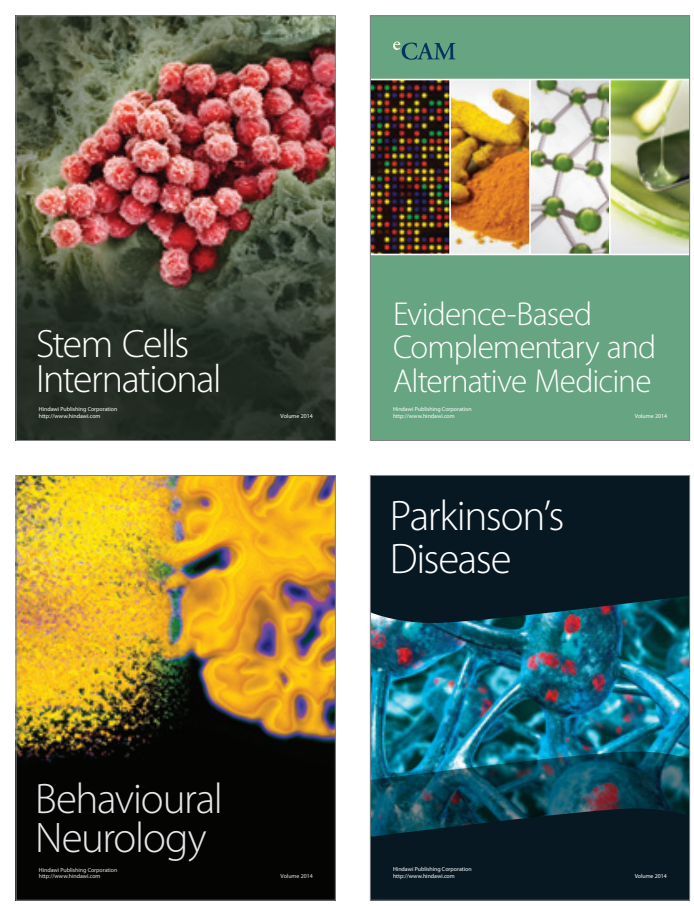

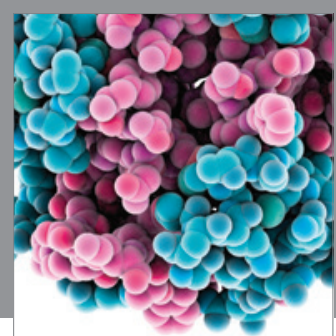

Journal of
Diabetes Research

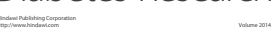

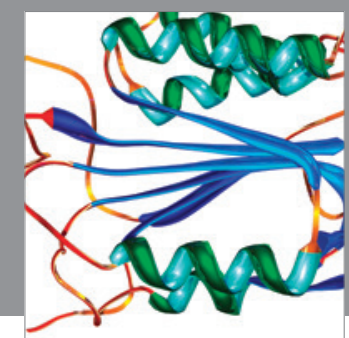

Disease Markers
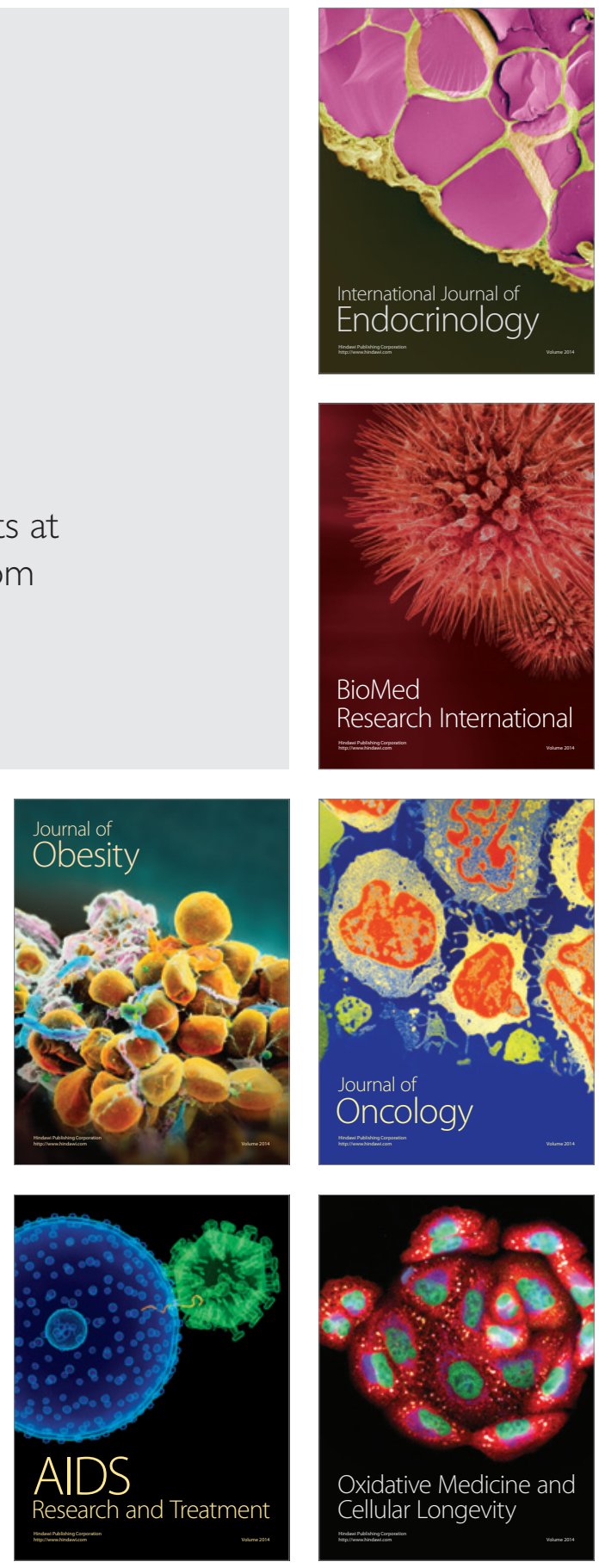